\title{
Adaptive Neural Stabilizing Controller for a Class of Mismatched Uncertain Nonlinear Systems by State and Output Feedback
}

\author{
Mohammad Mehdi Arefi, Mohammad Reza Jahed-Motlagh, and Hamid Reza Karimi, Senior Member, IEEE
}

\section{INTRODUCTION}

$\mathbf{R}$ OBUST control of nonlinear uncertain systems is one of the most important topics in both theoretical and practical aspects of control systems, having attracted a lot of devotion during the last three decades. Consequently, different methods relevant to stabilization of nonlinear dynamical systems with significant uncertainties have been presented in [1] and [2] and the references therein.

There are two main limitations related to uncertainties. The first problem is that several methods are constructed based on the so-called matching condition [3], [4]. To overcome this limitation, some methodologies for controlling uncertain nonlinear systems without matching condition have been presented [5], [6]. The second difficulty is the triangularity assumption, which is less restrictive than the matching

Manuscript received February 26, 2014; revised May 27, 2014, August 9, 2014, and September 2, 2014; accepted September 4, 2014. Date of publication September 26, 2014; date of current version July 15, 2015. This paper was recommended by Associate Editor P. Shi.

M. M. Arefi is with the Department of Power and Control Engineering, School of Electrical and Computer Engineering, Shiraz University, Shiraz 71348-51154, Iran (e-mail: arefi@ shirazu.ac.ir).

M. R. Jahed-Motlagh is with the Computer Engineering Department, Iran University of Science and Technology, Tehran 16846-13114, Iran (e-mail: jahedmr@iust.ac.ir).

H. R. Karimi is with the Department of Engineering, Faculty of Engineering and Science, University of Agder, Grimstad N-4898, Norway (e-mail: hamid.r.karimi@uia.no).

Color versions of one or more of the figures in this paper are available online. condition [3]. The design process with the triangularity condition affords the possibility of applying the designed controller to a broader range of uncertainties; however, this condition is also restrictive in many practical applications. Different methods have been used to control uncertain systems with mismatched uncertainties [7], [8]. Considering mismatched uncertainties is a challenging problem since it may degrade the system performance. These uncertainties can be seen in some real applications as stated in [9] and [10].

Variable structure control or sliding mode control (SMC) is one of the most powerful tools for stabilization and tracking problems [11]-[13]. In [14] and [15], applications of SMC are extensively provided. Recently, several techniques have been presented to control uncertain systems in the absence of triangularity assumption [16]-[18]. For example, in [16], an algorithm is presented to determine the projection matrix that minimizes mismatched perturbation. In addition, in [17], an adaptive integral SMC based on passivity is used to stabilize both parametric and nonparametric mismatched uncertainties. Moreover, it is assumed that the upper bounds of parameters, matched and mismatched uncertainties are known. Stabilization of mismatched uncertain systems based on Lyapunov stability theory and integral SMC for the known upper bound of mismatched uncertainties is addressed in [19].

In [18], a method for controlling linear mismatched uncertain systems by decomposition of uncertainties and matching condition assumption is presented. Furthermore, the tracking problem for a class of single-input single-output mismatched systems using multiple-surface sliding is proposed in [20]. Integral SMC for stabilization of a class of multiple-input multiple-output (MIMO) systems is studied in [21]. In addition, based on invariance condition, an SMC design is reported for large-scale systems with mismatched uncertainties [22].

To investigate asymptotic stability of time-delay MIMO systems, an SMC for regulation problems is presented in [23]. Besides, to achieve asymptotic stability of a class of MIMO systems using linear matrix inequality (LMI), a sliding surface is designed in [24]. In [25] and [26], SMC and LMI methods are utilized to achieve quadratic stability of dynamical uncertain systems using Lyapunov stability theory. Furthermore, using the same assumptions in [24]-[26], sliding, adaptive fuzzy sliding, and adaptive fuzzy sliding mode controllers are presented in [18], [27], and [28]. In [29], a delay-dependent exponential H8 synchronization for uncertain neural networks (NNs) with mixed time delays utilizing LMIs was reported. 
Adaptive output feedback control for tracking problems without knowing mismatched uncertainties is reported in [30]. However, asymptotic stability is not assured in this paper. It is noticeable that in the most cited works using SMC, it is required knowing the upper bound of unknown uncertainties [10], [31], [32]. In recent years, considerable efforts have been made to design mismatched control systems with no assumption on the bound of uncertainties [33], [34]. These controllers are applicable for systems that satisfy $n \leq 2 m$ condition, where the system order is $n$ and $m$ is the number of inputs. Recently, for the systems with $n>2 m$ an adaptive SMC is presented [35]. More recently, a block backstepping controller for a class of perturbed nonlinear systems with $m$ blocks is proposed using an adaptive mechanism [36]. The design procedure is such that the least upper bound of perturbations except that of the input channel is not required during the design process. $\mathrm{NN}$ and fuzzy logic as universal approximator can be used as an alternative approach to approximate unknown uncertainties [37]-[41]. Furthermore, radial basis functions NN (RBFNN) is often used in practical applications due to simple structure and nice approximation properties [40], [42]-[44]. In addition, among various model-based fuzzy control approaches, in particular, the method based on Takagi-Sugeno (T-S) model is well-matched to the continuous and discrete model-based nonlinear control [45]-[49].

Although different methods are presented for controlling nonlinear systems, most of the previous results are based on the assumption that state variables of the system are available. If the system states are unavailable, these results are not applicable in practice and control techniques using estimated states are then required [46], [47], [50]-[52]. For example, the problem of robust $H_{\infty}$ output feedback control for a class of networked nonlinear systems is investigated in [46]. In [47], the dynamic output feedback control problem is investigated for discrete-time T-S fuzzy systems with time-varying delays. In addition, output feedback controller for discrete-time Markovian jump repeated scalar nonlinear systems is presented in [50]. The problem of output-feedback-based $\mathrm{H} 8$ control for active suspension systems with control delay has been reported in [53]. Furthermore, in [54], static output-feedback control under information structure constraints is presented.

In this paper, two adaptive NN stabilization controllers for a broad class of nonlinear systems with mismatched uncertainties are proposed. The first controller is based on state-feedback, while the second one is designed based on estimated states. Moreover, the proposed scheme possesses the following features.

1) This paper successfully extends adaptive neural controller for nonlinear systems with mismatched uncertainties. In this approach, the upper bound of uncertainties is not required to be known in advance. This upper bound is approximated using NN through an adaptation mechanism.

2) An observer is designed to estimate unmeasurable states for the control purpose.

3) By using a robustifying term in the control signal, the effects of approximation error in NN and external disturbance is compensated.
The rest of this paper is organized as follows. The problem formulation is given in Section II. In Section III, we present a procedure to design adaptive $\mathrm{NN}$ controller using statefeedback method. Moreover, the stability results are provided in this section. Observer-based adaptive NN control for uncertain systems and its stability is investigated in Section IV. In Section V, simulation results are presented to show the proficiency of the proposed scheme. Finally, the conclusion is given in Section VI.

\section{PROBLEM Formulation}

Consider the following nonlinear system with mismatched uncertainties and external matched disturbance:

$$
\dot{\mathbf{x}}=\mathbf{A x}+\mathbf{f}(\mathbf{x})+\mathbf{B}[\mathbf{u}+\mathbf{d}]
$$

where $\mathbf{x}=\left[x_{1}, \ldots, x_{n}\right]^{\mathrm{T}} \in \mathfrak{R}^{n}$ is the vector of system states, $\mathbf{u}=\left[u_{1}, \ldots, u_{m}\right]^{\mathrm{T}} \in \mathfrak{R}^{m}$ is the vector of system inputs, $\mathbf{f}(\mathbf{x})=\left[f_{1}(\mathbf{x}), \ldots, f_{n}(\mathbf{x})\right]^{\mathrm{T}}$ is the vector of smooth nonlinear mismatched uncertain functions, and $\mathbf{d} \in \mathfrak{R}^{m}$ is the vector of external disturbance. Moreover, A, $\mathbf{B}$ are system matrices with appropriate dimensions. The goal is to design a stabilizing controller so that the states of the closed-loop system will be remained stable.

In this paper, the Gaussian $\mathrm{RBF}$ will be employed to approximate a nonlinear function $h():. \mathfrak{R}^{n} \rightarrow \mathfrak{R}$ as follows:

$$
\hat{h}(\mathbf{z})=\boldsymbol{\Theta} \xi(\mathbf{z})
$$

where $\mathbf{z} \in \Omega_{\mathrm{NN}} \subset \mathfrak{R}^{n}$ is the input vector, $\boldsymbol{\Theta}=$ $\left[\theta_{1} \theta_{2} \cdots \theta_{l}\right] \in \mathfrak{R}^{l}$ is the weight vector, $l$ is the number of nodes, and $\xi(\mathbf{z})=\left[\xi_{1}(\mathbf{z}), \ldots, \xi_{l}(\mathbf{z})\right]^{\mathrm{T}}$ is the basis function determined priory, and commonly chosen as Gaussian functions

$$
\xi_{i}(\mathbf{z})=\exp \left(-\frac{\left\|\mathbf{z}-\boldsymbol{\mu}_{i}\right\|^{2}}{\eta_{i}^{2}}\right), \quad i=1,2, \ldots, l
$$

where $\mu_{i}=\left[\mu_{i 1}, \mu_{i 1}, \ldots, \mu_{\text {in }}\right]^{\mathrm{T}}$ and $\eta_{i}$ are the center and width of Gaussian functions, respectively. By choosing enough nodes, NNs can approximate function $h($.) with an arbitrary accuracy over a compact region $\Omega_{\mathrm{NN}} \subset \Re^{n}$

$$
h(\mathbf{z})=\boldsymbol{\Theta}^{*} \xi(\mathbf{z})+\delta, \quad \forall \mathbf{z} \in \Omega_{\mathrm{NN}}
$$

where $\delta$ is the approximation error of NN. The optimal weight vector $\boldsymbol{\Theta}^{*}$ is defined as

$$
\boldsymbol{\Theta}^{*}=\arg \min _{\boldsymbol{\Theta} \in \Re^{l}}\left\{\sup _{\mathbf{z} \in \Omega_{\mathrm{NN}}}|h(\mathbf{z})-\hat{h}(\mathbf{z})|\right\} .
$$

Remark 1: It is noting that this structure of RBFNN is $n-l-1$ ( $n$ input variables, $l$ hidden neurons and a singleoutput neuron) can reduce the complexity of computational problems so as to speed-up the learning process, especially for accomplishing the function approximation and model identification, for instance.

Now, suppose $\{z(t) ; t=1, \ldots, s\}$ is a set of data samples. According to the structure of RBFNN, the computation cost is $n \times l \times s$.

Assumption 1: The pair (A, B) is controllable. This assumption assures that a gain matrix $\mathbf{K}_{c}$ exists such that the 
characteristic polynomial of $\mathbf{A}-\mathbf{B} \mathbf{K}_{c}^{\mathrm{T}}$ is Hurwitz. This guarantees for a given positive definite matrix $\mathbf{Q}$, there exists a positive-definite solution $\mathbf{P}$ for the following matrix equation:

$$
\left(\mathbf{A}-\mathbf{B} \mathbf{K}_{\mathrm{c}}^{\mathrm{T}}\right)^{\mathrm{T}} \mathbf{P}+\mathbf{P}\left(\mathbf{A}-\mathbf{B} \mathbf{K}_{\mathrm{c}}^{\mathrm{T}}\right)+\mathbf{Q}=\mathbf{0} .
$$

Assumption 2: The disturbance vector $\mathbf{d}(t)$ is bounded. In other words, the relation $\|\mathbf{d}\|<\delta_{d}$ is true, where $\delta_{d}$ is the upper bound of external disturbance vector.

\section{State-FeEdback Stabilizer Design}

In order to design state-feedback stabilizer, the control input is selected as

$$
\mathbf{u}=-\mathbf{K}_{c}^{\mathrm{T}} \mathbf{x}-\mathbf{u}_{\mathrm{adp}}-\mathbf{u}_{R}-\mathbf{u}_{d}
$$

where $\mathbf{u}_{\text {adp }}$ is an adaptive part to compensate the effects of mismatched uncertainties. In addition, $\mathbf{u}_{R}$ and $\mathbf{u}_{d}$ are designed to compensate the effects of NN approximation error and external disturbance, respectively.

Define the functions $\eta^{T}$ and $g(\mathbf{x})$ as follows:

$$
\begin{aligned}
\eta^{\mathrm{T}}(\mathbf{x}) & =\mathbf{x}^{\mathrm{T}} \mathbf{P B} \\
g(\mathbf{x}) & =\frac{\mathbf{x}^{\mathrm{T}} \mathbf{P f}(\mathbf{x})}{\|\boldsymbol{\eta}(\mathbf{x})\|^{2}} .
\end{aligned}
$$

By using universal approximation property of NNs, $g(\mathbf{x})$ can be approximated as

$$
\hat{g}(\mathbf{x})=\boldsymbol{\Theta} \xi(\mathbf{x}) .
$$

It is assumed that $\mathbf{x}$ and $\boldsymbol{\Theta}$ belong to compact sets $U_{x}$ and $\Omega$, respectively, where defined as

$$
\begin{aligned}
U_{x} & =\left\{\mathbf{x} \in \Re^{n}:\|\mathbf{x}\| \leq M_{1}\right\} \\
\Omega & =\left\{\boldsymbol{\Theta} \in \mathfrak{R}^{l}:\|\boldsymbol{\Theta}\| \leq M_{2}\right\}
\end{aligned}
$$

and $M_{1}$ and $M_{2}$ are design parameters.

Assumption 3: Suppose that the function $g(\mathbf{x})$ is bounded in $U_{x}$. This assumption states that if the degree of uncertainty $\mathbf{f}(\mathbf{x})$ is equal or greater than state $\mathbf{x}$, i.e., $(\|\mathbf{f}(\mathbf{x})\| /\|\mathbf{x}\|)$ exists in $U_{x}$, then $g(\mathbf{x})$ is bounded in this compact set. This assumption is realistic because the degree of denominator of $g(\mathbf{x})$ is always equal to two.

The optimal parameter vector $\boldsymbol{\Theta}^{*}$ is defined as

$$
\boldsymbol{\Theta}^{*}=\arg \min _{\boldsymbol{\Theta} \in \Omega}\left\{\sup _{\mathbf{x} \in U_{x}}|g(\mathbf{x})-\hat{g}(\mathbf{x} \mid \boldsymbol{\Theta})|\right\} \text {. }
$$

Furthermore, NN approximation error is represented as

$$
\delta_{n}(\mathbf{x})=g(\mathbf{x})-\hat{g}\left(\mathbf{x} \mid \Theta^{*}\right) .
$$

Here, $\boldsymbol{\Theta}$ denotes the estimation of $\boldsymbol{\Theta}^{*}$. In addition, the estimation error is defined as $\tilde{\boldsymbol{\Theta}}=\boldsymbol{\Theta}-\boldsymbol{\Theta}^{*}$. Consider the adaptive neural controller (7), where

$$
\begin{aligned}
\mathbf{u}_{\mathrm{adp}} & =\eta(\mathbf{x}) \boldsymbol{\Theta} \xi(\mathbf{x}) \\
\mathbf{u}_{R} & =k_{1} \boldsymbol{\eta}(\mathbf{x})
\end{aligned}
$$

and

$$
\mathbf{u}_{d}=k_{2} \operatorname{sgn}\left(\mathbf{B}^{\mathrm{T}} \mathbf{P x}\right)
$$

The adaptation law for updating the estimation vector $\boldsymbol{\Theta}$ is

$$
\dot{\boldsymbol{\Theta}}=\gamma\|\eta\|^{2} \xi^{\mathrm{T}}(\mathbf{x})
$$

where $\gamma>0$ is an adaptation gain to be designed.

Lemma 1 (Barbalat Lemma): If $\zeta(t)$ is a real function of real variable $t$ which is defined and uniformly continuous for $t \geq t_{0}, \quad t_{0} \in \Re^{+}$, and if the limit of integral $\int_{t_{0}}^{t} \zeta(\tau) d \tau$ as $t$ tends to infinity, exists and is a finite number, then $\lim \zeta(t)=0[3]$.

Theorem 1: For the system (1), consider control law (7) with (14)-(16) and adaptation law (17). If Assumptions 1-3 are satisfied, then the proposed adaptive neural controller guarantees

$$
\lim _{t \rightarrow \infty} \mathbf{x}(t)=\mathbf{0} \text {. }
$$

Proof: Consider the following continuous differentiable function as a Lyapunov candidate:

$$
\mathrm{V}=\frac{1}{2} \mathbf{x}^{\mathbf{T}} \mathbf{P} \mathbf{x}+\frac{1}{2 \gamma} \tilde{\boldsymbol{\Theta}} \tilde{\boldsymbol{\Theta}}^{\mathrm{T}} .
$$

The derivative of $\mathrm{V}$ is

$$
\dot{\mathrm{V}}=\frac{1}{2} \dot{\mathbf{x}}^{\mathrm{T}} \mathbf{P} \mathbf{x}+\frac{1}{2} \mathbf{x}^{\mathrm{T}} \mathbf{P} \dot{\mathbf{x}}+\frac{1}{\gamma} \tilde{\boldsymbol{\Theta}} \dot{\tilde{\Theta}}^{\mathrm{T}} .
$$

Substituting (1), (7), and (14) in (19) gives

$$
\begin{aligned}
\dot{\mathrm{V}}= & \frac{1}{2} \mathbf{x}^{\mathrm{T}}\left[\left(\mathbf{A}-\mathbf{B} \mathbf{K}_{c}^{\mathrm{T}}\right)^{\mathrm{T}} \mathbf{P}+\mathbf{P}\left(\mathbf{A}-\mathbf{B} \mathbf{K}_{c}^{\mathrm{T}}\right)\right] \mathbf{x}+\mathbf{x}^{\mathrm{T}} \mathbf{P} \mathbf{f}(\mathbf{x}) \\
& +\mathbf{x}^{\mathrm{T}} \mathbf{P B}\left[-\boldsymbol{\eta}(\mathbf{x}) \boldsymbol{\Theta} \xi(\mathbf{x})-\mathbf{u}_{R}-\mathbf{u}_{d}+\mathbf{d}\right]+\frac{1}{\gamma} \tilde{\boldsymbol{\Theta}} \dot{\tilde{\Theta}}^{\mathrm{T}} .
\end{aligned}
$$

Then, by using (6), one can write

$$
\begin{aligned}
\dot{\mathrm{V}}= & -\frac{1}{2} \mathbf{x}^{\mathrm{T}} \mathbf{Q} \mathbf{x}+\mathbf{x}^{\mathrm{T}} \mathbf{P f}(\mathbf{x})+\mathbf{x}^{\mathrm{T}} \mathbf{P B} \\
& {\left[-\eta(\mathbf{x}) \boldsymbol{\Theta} \xi(\mathbf{x})-\mathbf{u}_{R}-\mathbf{u}_{d}+\mathbf{d}\right]+\frac{1}{\gamma} \tilde{\boldsymbol{\Theta}} \dot{\tilde{\mathbf{\Theta}}}^{\mathrm{T}} }
\end{aligned}
$$

and using (9), we have

$$
\begin{aligned}
\dot{\mathrm{V}}= & -\frac{1}{2} \mathbf{x}^{\mathrm{T}} \mathbf{Q} \mathbf{x}+\|\boldsymbol{\eta}(\mathbf{x})\|^{2} g(\mathbf{x})+\mathbf{x}^{\mathrm{T}} \mathbf{P B} \\
& {\left[-\eta(\mathbf{x}) \Theta \xi(\mathbf{x})-\mathbf{u}_{R}-\mathbf{u}_{d}+\mathbf{d}\right]+\frac{1}{\gamma} \tilde{\mathbf{\Theta}} \dot{\tilde{\Theta}}^{\mathrm{T}} . }
\end{aligned}
$$

By using (8), (10), and (13) we can write

$$
\begin{aligned}
\dot{\mathrm{V}}= & -\frac{1}{2} \mathbf{x}^{\mathrm{T}} \mathbf{Q} \mathbf{x}+\|\boldsymbol{\eta}(\mathbf{x})\|^{2}\left(\boldsymbol{\Theta}^{*} \xi(\mathbf{x})-\boldsymbol{\Theta} \xi(\mathbf{x})+\delta_{n}\right) \\
& +\mathbf{x}^{\mathrm{T}} \mathbf{P B}\left[-\mathbf{u}_{R}-\mathbf{u}_{d}+\mathbf{d}\right]+\frac{1}{\gamma} \tilde{\boldsymbol{\Theta}} \dot{\tilde{\Theta}}^{\mathrm{T}} .
\end{aligned}
$$

Since $\tilde{\boldsymbol{\Theta}}=\boldsymbol{\Theta}-\boldsymbol{\Theta}^{*}$, (23) can be written as

$$
\begin{aligned}
\dot{\mathrm{V}}= & -\frac{1}{2} \mathbf{x}^{\mathrm{T}} \mathbf{Q} \mathbf{x}+\|\boldsymbol{\eta}(\mathbf{x})\|^{2}\left(-\tilde{\boldsymbol{\Theta}} \xi(\mathbf{x})+\delta_{n}\right)+\mathbf{x}^{\mathrm{T}} \mathbf{P B} \\
& {\left[-\mathbf{u}_{R}-\mathbf{u}_{d}+\mathbf{d}\right]+\frac{1}{\gamma} \tilde{\boldsymbol{\Theta}} \dot{\tilde{\Theta}}^{\mathrm{T}} . }
\end{aligned}
$$

In the following, by substituting adaptation law (17) in (24), we have:

$$
\dot{\mathrm{V}}=-\frac{1}{2} \mathbf{x}^{\mathrm{T}} \mathbf{Q} \mathbf{x}+\|\boldsymbol{\eta}(\mathbf{x})\|^{2} \delta_{n}+\mathbf{x}^{\mathrm{T}} \mathbf{P B}\left[-\mathbf{u}_{R}-\mathbf{u}_{d}+\mathbf{d}\right]
$$


then using (15), (16), and Assumption 2 yields

$\dot{\mathrm{V}} \leq-\frac{1}{2} \mathbf{x}^{\mathrm{T}} \mathbf{Q} \mathbf{x}+\|\boldsymbol{\eta}(\mathbf{x})\|^{2}\left(\delta_{n}-k_{1}\right)+\left(\delta_{d}-k_{2}\right) \sum_{i=1}^{m}\left|\left(\mathbf{x}^{\mathrm{T}} \mathbf{P B}\right)_{i}\right|$.

Now, if $k_{1} \geq\left\|\delta_{n}\right\|$ and $k_{2} \geq \delta_{d}$, we have

$$
\dot{\mathrm{V}} \leq-\frac{1}{2} \mathbf{x}^{\mathrm{T}} \mathbf{Q} \mathbf{x}
$$

Finally, using Rayleigh inequality [55], $-\mathbf{x}^{\mathrm{T}} \mathbf{Q} \mathbf{x} \leq$ $-\lambda_{\min }(\mathbf{Q})\|\mathbf{x}\|^{2}$, one can write

$$
\dot{\mathrm{V}} \leq-\frac{1}{2} \lambda_{\min }(\mathbf{Q})\|\mathbf{x}\|^{2}
$$

By integrating from both sides with respect to time, (28) can be further written as

$$
\lambda_{\min }(\mathbf{Q}) \int_{t_{0}}^{t} \mathbf{x}^{\mathrm{T}}(\tau) \mathbf{x}(\tau) d \tau \leq V\left(t_{0}\right)-V(t)
$$

which implies

$$
\lim _{t \rightarrow \infty} \lambda_{\min }(\mathbf{Q}) \int_{t_{0}}^{t} \mathbf{x}^{\mathrm{T}}(\tau) \mathbf{x}(\tau) d \tau \leq V\left(t_{0}\right)-V(\infty)<\infty .
$$

Let $\psi(t)=\mathbf{z}^{\mathrm{T}}(t) \mathbf{z}(t)$. From (18) and (28), it is derived that $\|\mathbf{x}(t)\|$ and $\|\tilde{\boldsymbol{\Theta}}\|$ are bounded. From (1), since $\mathbf{x}(t)$ is bounded, it is concluded that $\dot{\mathbf{x}}(t)$ is bounded. Therefore, $\psi(t)$ is uniformly continuous and it is possible to apply the Barbalat lemma to the function $\psi(t)$ which guarantees $\lim _{t \rightarrow \infty} \psi(t)=0$, and consequently, it is deduced that $\lim _{t \rightarrow \infty}\|\mathbf{x}(t)\| \stackrel{t \rightarrow \infty}{=} 0$ which completes the proof.

Corollary 1: The weighs of NN ultimately converge to constant values.

Proof: According to Theorem 1, since $\lim _{t \rightarrow \infty}\|\mathbf{x}(t)\|=0$, using (8) and (17), one can conclude $\lim _{t \rightarrow \infty} \dot{\Theta}=\mathbf{0}$. Consequently, the adaptation parameters of NN approach to constant values.

Remark 2: The stability results in this paper are semi-global which means for any compact set, there exists a controller with neural approximation with sufficiently large hidden nodes such that when the initial states are within this set, all the closed-loop signals are bounded. In realistic applications due to computational problem, the hidden nodes usually cannot be chosen too large which implies that the neural system approximation ability is limited and some constraints on compact set are necessary to guarantee such an approximation.

Remark 3: Since the control law (7) contains the sign function, direct application of such control may result in chattering caused by the signal discontinuity. In order to remedy the chattering phenomenon, the sign function is replaced by saturation function with a boundary layer $\phi_{i}, i=1,2, \ldots, p$ of the form

$$
\operatorname{sat}\left(\frac{\left(\mathbf{x}^{\mathrm{T}} \mathbf{P B}\right)_{i}}{\phi_{i}}\right)= \begin{cases}\operatorname{sgn}\left(\frac{\left(\mathbf{x}^{\mathrm{T}} \mathbf{P B}\right)_{i}}{\phi_{i}}\right) & \left|\frac{\left(\mathbf{x}^{\mathrm{T}} \mathbf{P B}\right)_{i}}{\phi_{i}}\right|>1 \\ \frac{\left(\mathbf{x}^{\mathrm{T}} \mathbf{P B}\right)_{i}}{\phi_{i}} & \left|\frac{\left(\mathbf{x}^{\mathrm{T}} \mathbf{P B}\right)_{i}}{\phi_{i}}\right| \leq 1 .\end{cases}
$$

The stability result is then semi-globally uniformly ultimately bounded.

\section{OBSERVER-BASEd StabiLIZER DESIGN}

In a real dynamic system, the states of the systems may not be available for measurement. In this case, the results in Section III are not applicable in practice and the NN-based adaptive control using estimated states is then required. Let us consider the following mismatched uncertain system:

$$
\begin{aligned}
& \dot{\mathbf{x}}=\mathbf{A x}+\mathbf{f}(\mathbf{x})+\mathbf{B}[\mathbf{u}+\mathbf{d}] \\
& \mathbf{y}=\mathbf{C}^{\mathrm{T}} \mathbf{x}
\end{aligned}
$$

where $\mathbf{y} \in \Re^{m}$ are the measurable outputs of the system. The control objective is that the system states are regulated by using available outputs. Since the state vector $\mathbf{x}$ is assumed to be immeasurable, it cannot be used in the controller design. Therefore, an observer should be designed to estimate the immeasurable states.

Assumption 4: The pair $\left(\mathbf{A}, \mathbf{C}^{\mathrm{T}}\right)$ is observable. This assumption assures that a gain matrix $\mathbf{K}_{o}$ exists such that the characteristic polynomial of $\mathbf{A}-\mathbf{K}_{o} \mathbf{C}^{\mathrm{T}}$ is Hurwitz.

Assumption 5: For the given positive-definite matrix $\mathbf{Q}_{1}$, there exists positive-definite solution $\mathbf{P}_{1}$ for the matrix equations

$$
\begin{aligned}
\left(\mathbf{A}-\mathbf{K}_{o} \mathbf{C}^{\mathrm{T}}\right)^{\mathrm{T}} \mathbf{P}_{1}+\mathbf{P}_{1}\left(\mathbf{A}-\mathbf{K}_{o} \mathbf{C}^{\mathrm{T}}\right)+\mathbf{Q}_{1}=\mathbf{0} \\
\mathbf{P}_{1} \mathbf{B}=\mathbf{C} .
\end{aligned}
$$

Remark 4: If $\mathbf{K}_{o}$ can be chosen such that the triple $\left(\mathbf{A}-\mathbf{K}_{o} \mathbf{C}^{\mathrm{T}}, \mathbf{B}, \mathbf{C}^{\mathrm{T}}\right)$ is strictly positive real (SPR), one can use Kalman-Yakubovich-Popov lemma [15], which guarantees the existence of positive definite symmetric matrices $\mathbf{P}_{1}$ and $\mathbf{Q}_{1}$ in (33).

Theorem 2 [56]: Suppose the matrix A is Hurwitz. Then the transfer function $\mathbf{G}(s)=\mathbf{C}(\mathbf{s} I-\mathbf{A})^{-1} \mathbf{B}$ is SPR if and only if

$$
\tilde{\mathbf{R}}:=-\mathbf{C A B}-(\mathbf{C A B})^{\mathrm{T}}>\mathbf{0}
$$

and the Hamiltonian matrix

$$
\mathbf{M}=\left(\begin{array}{cc}
\mathbf{A}+\mathbf{A} \mathbf{B} \tilde{\mathbf{R}}^{-1} \mathbf{C A} & \mathbf{A} \mathbf{B} \tilde{\mathbf{R}}^{-1} \mathbf{B}^{\mathrm{T}} \mathbf{A}^{\mathrm{T}} \\
-\mathbf{A}^{\mathrm{T}} \mathbf{C}^{\mathrm{T}} \tilde{\mathbf{R}}^{-1} \mathbf{C A}-\mathbf{A}^{\mathrm{T}}-\mathbf{A}^{\mathrm{T}} \mathbf{C}^{\mathrm{T}} \tilde{\mathbf{R}}^{-1} \mathbf{B}^{\mathrm{T}} \mathbf{A}^{\mathrm{T}}
\end{array}\right)
$$

has no eigenvalues on the imaginary axis except at zero where it has exactly $2 m$ eigenvalues where $m$ is the number of inputs.

Consider the following observer:

$$
\begin{aligned}
\dot{\hat{\mathbf{x}}} & =\mathbf{A} \hat{\mathbf{x}}-\mathbf{B} \mathbf{K}_{c}^{\mathrm{T}} \hat{\mathbf{x}}+\mathbf{K}_{o}(\mathbf{y}-\hat{\mathbf{y}}) \\
\hat{\mathbf{y}} & =\mathbf{C}^{\mathrm{T}} \hat{\mathbf{x}}
\end{aligned}
$$

where $\hat{\mathbf{x}}$ is the estimation of vector $\mathbf{x}$. Define the observation error as $\tilde{\mathbf{x}}=\mathbf{x}-\hat{\mathbf{x}}$. Then, by using (32) and (36) one can write

$$
\begin{aligned}
& \dot{\tilde{\mathbf{x}}}=\left(\mathbf{A}-\mathbf{K}_{o} \mathbf{C}^{\mathrm{T}}\right) \tilde{\mathbf{x}}+\mathbf{B}\left[\mathbf{K}_{c}^{\mathrm{T}} \hat{\mathbf{x}}+\mathbf{u}+\mathbf{d}\right]+\mathbf{f}(\mathbf{x}) \\
& \tilde{\mathbf{y}}=\mathbf{C}^{\mathrm{T}} \tilde{\mathbf{x}} .
\end{aligned}
$$

Define function $\eta_{1}^{\mathrm{T}}$ as

$$
\eta_{1}^{\mathrm{T}}(\tilde{\mathbf{x}})=\tilde{\mathbf{x}}^{\mathrm{T}} \mathbf{P}_{1} \mathbf{B}
$$

Meanwhile, $g_{1}(\mathbf{x}, \tilde{\mathbf{x}})$ is defined as follows:

$$
g_{1}(\mathbf{x}, \tilde{\mathbf{x}})=\frac{\tilde{\mathbf{x}}^{\mathrm{T}} \mathbf{P}_{1} \mathbf{f}(\mathbf{x})}{\left\|\eta_{1}(\tilde{\mathbf{x}})\right\|^{2}}
$$




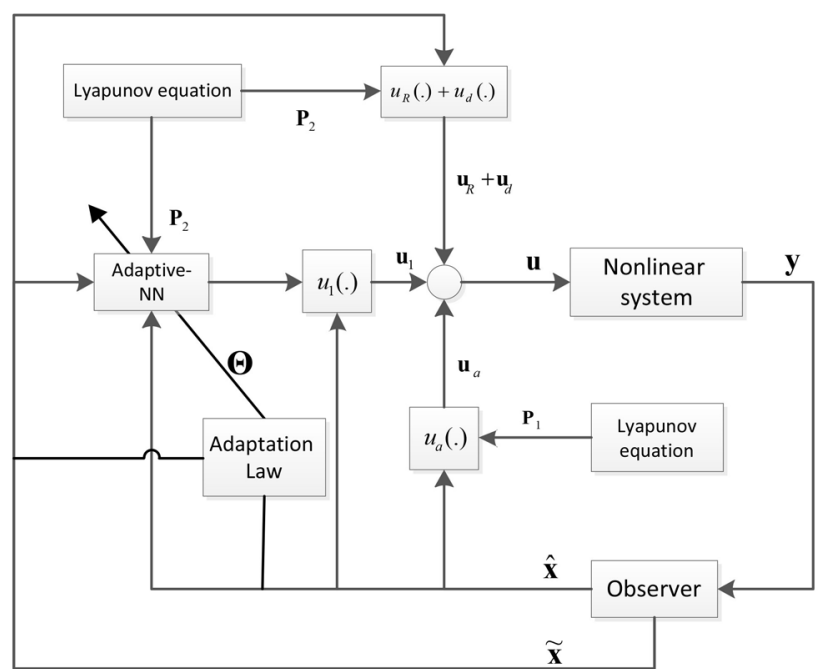

Fig. 1. Overall design procedure of the observer-based adaptive-neural stabilizer for mismatched uncertain systems.

It is assumed that $\hat{\mathbf{x}}$ belongs to compact sets $\mathrm{U}_{\hat{\mathbf{x}}}$ which is defined as

$$
\mathrm{U}_{\hat{\mathbf{x}}}=\left\{\hat{\mathbf{x}} \in \Re^{n}:\|\hat{\mathbf{x}}\| \leq \mathrm{M}_{3}\right\} .
$$

Assumption 6: Suppose that the function $g_{1}(\mathbf{x}, \tilde{\mathbf{x}})$ is bounded in $U_{x}$ and $\mathrm{U}_{\hat{\mathbf{x}}}$.

Similar to the previous design, by using an RBFNN, $g_{1}(\mathbf{x}, \tilde{\mathbf{x}})$ can be approximated as

$$
\hat{g}_{1}(\hat{\mathbf{x}})=\boldsymbol{\Theta}_{1} \xi(\hat{\mathbf{x}}) .
$$

Then the optimal parameter vector $\boldsymbol{\Theta}_{1}^{*}$ is defined as

$$
\boldsymbol{\Theta}_{1}^{*}=\arg \min _{\boldsymbol{\Theta}_{1} \in \Omega}\left\{\sup _{\mathbf{x} \in U_{x}, \hat{\mathbf{x}} \in U_{\hat{\mathbf{x}}}}\left|g_{1}(\mathbf{x}, \tilde{\mathbf{x}})-\hat{g}_{1}\left(\hat{\mathbf{x}} \mid \boldsymbol{\Theta}_{1}\right)\right|\right\} \text {. }
$$

Furthermore, NN approximation error is represented as follows:

$$
\delta_{n 1}(\mathbf{x}, \hat{\mathbf{x}})=g_{1}(\mathbf{x}, \tilde{\mathbf{x}})-\hat{g}_{1}\left(\hat{\mathbf{x}} \mid \Theta_{1}^{*}\right)
$$

where $\boldsymbol{\Theta}_{1}$ is the estimated value of vector $\boldsymbol{\Theta}_{1}^{*}$. In addition, the estimation error is defined as $\tilde{\boldsymbol{\Theta}}_{1}=\boldsymbol{\Theta}_{1}-\boldsymbol{\Theta}_{1}^{*}$. Consider the following adaptive neural controller:

$$
\mathbf{u}=-\mathbf{K}_{c}^{\mathrm{T}} \hat{\mathbf{x}}-\boldsymbol{\eta}_{1}(\tilde{\mathbf{x}}) \boldsymbol{\Theta}_{1} \xi(\hat{\mathbf{x}})-\mathbf{u}_{a}-\mathbf{u}_{R}-\mathbf{u}_{d}
$$

where

$$
\begin{aligned}
\mathbf{u}_{a} & =K_{o}^{\mathrm{T}} \mathbf{P} \hat{\mathbf{x}} \\
\mathbf{u}_{R} & =k_{1} \boldsymbol{\eta}_{1}(\tilde{\mathbf{x}})
\end{aligned}
$$

and

$$
\mathbf{u}_{d}=k_{2} \operatorname{sgn}\left(\mathbf{B}^{\mathrm{T}} \mathbf{P}_{1} \tilde{\mathbf{x}}\right) .
$$

In (44), $\mathbf{u}_{a}$ is a feedback of estimated states, $\mathbf{u}_{R}$ and $\mathbf{u}_{d}$ are the controllers to compensate $\mathrm{NN}$ approximation error and external disturbances, respectively. The adaptation law for updating (44) is proposed as

$$
\dot{\boldsymbol{\Theta}}_{1}=\gamma\left\|\boldsymbol{\eta}_{1}(\tilde{\mathbf{x}})\right\|^{2} \xi^{\mathrm{T}}(\hat{\mathbf{x}})
$$

where $\gamma>0$ is the adaptation gain and should be selected by the designer. Fig. 1 shows the overall scheme of the observer-based adaptive neural stabilizing controller for mismatched uncertain nonlinear system.

The main property of adaptive-NN stabilizer scheme is summarized in the following theorem.

Theorem 3: For the system (32), consider controllers (44)-(47) and the adaptation law (48). If Assumptions 1, 2, 4-6 are satisfied, then the proposed adaptive-NN guarantees

$$
\lim _{t \rightarrow \infty} \mathbf{x}(t)=\mathbf{0} .
$$

Proof: Consider the Lyapunov function candidate

$$
\mathrm{V}=\frac{1}{2} \hat{\mathbf{x}}^{\mathrm{T}} \mathbf{P} \hat{\mathbf{x}}+\frac{1}{2} \tilde{\mathbf{x}}^{\mathrm{T}} \mathbf{P}_{1} \tilde{\mathbf{x}}+\frac{1}{2 \gamma} \tilde{\boldsymbol{\Theta}}_{1} \tilde{\Theta}_{1}^{\mathrm{T}} .
$$

The derivative of (49) is as

$$
\begin{aligned}
\dot{\mathrm{V}}= & \frac{1}{2} \dot{\hat{\mathbf{x}}}^{\mathrm{T}} \mathbf{P} \hat{\mathbf{x}}+\frac{1}{2} \hat{\mathbf{x}}^{\mathrm{T}} \mathbf{P} \dot{\hat{\mathbf{x}}}+\frac{1}{2} \dot{\tilde{\mathbf{x}}}^{\mathrm{T}} \mathbf{P}_{1} \tilde{\mathbf{x}}+\frac{1}{2} \tilde{\mathbf{x}}^{\mathrm{T}} \mathbf{P}_{1} \dot{\tilde{\mathbf{x}}} \\
& +\frac{1}{\gamma} \tilde{\boldsymbol{\Theta}}_{1} \dot{\tilde{\mathbf{\Theta}}}_{1}^{\mathrm{T}} \frac{n !}{r !(n-r) !} .
\end{aligned}
$$

Substituting (36) and (37) in (50) yields

$$
\begin{aligned}
\dot{\mathrm{V}}= & \frac{1}{2} \hat{\mathbf{x}}^{\mathrm{T}}\left[\left(\mathbf{A}-\mathbf{B} \mathbf{K}_{c}^{\mathrm{T}}\right)^{\mathrm{T}} \mathbf{P}+\mathbf{P}\left(\mathbf{A}-\mathbf{B} \mathbf{K}_{c}^{\mathrm{T}}\right)\right] \hat{\mathbf{x}}+\hat{\mathbf{x}}^{\mathrm{T}} \mathbf{P} \mathbf{K}_{o} \mathbf{C}^{\mathrm{T}} \tilde{\mathbf{x}} \\
& +\frac{1}{2} \tilde{\mathbf{x}}^{\mathrm{T}}\left[\left(\mathbf{A}-\mathbf{K}_{o} \mathbf{C}^{\mathrm{T}}\right)^{\mathrm{T}} \mathbf{P}_{1}+\mathbf{P}_{1}\left(\mathbf{A}-\mathbf{K}_{o} \mathbf{C}^{\mathrm{T}}\right)\right] \tilde{\mathbf{x}} \\
& +\tilde{\mathbf{x}}^{\mathrm{T}} \mathbf{P}_{1} \mathbf{B}\left[\mathbf{K}_{c}^{\mathrm{T}} \hat{\mathbf{x}}+\mathbf{u}+\mathbf{d}\right]+\tilde{\mathbf{x}}^{\mathrm{T}} \mathbf{P}_{1} \mathbf{f}(\mathbf{x})+\frac{1}{\gamma} \tilde{\boldsymbol{\Theta}}_{1} \dot{\tilde{\Theta}}_{1}^{\mathrm{T}} .
\end{aligned}
$$

Then using (6) and (33), gives

$$
\begin{aligned}
\dot{\mathrm{V}}= & -\frac{1}{2} \hat{\mathbf{x}}^{\mathrm{T}} Q \hat{\mathbf{x}}-\frac{1}{2} \tilde{\mathbf{x}}^{\mathrm{T}} \mathbf{Q}_{1} \tilde{\mathbf{x}}+\hat{\mathbf{x}}^{\mathrm{T}} \mathbf{P}_{1} \mathbf{K}_{o} \mathbf{C}^{\mathrm{T}} \tilde{\mathbf{x}} \\
& +\tilde{\mathbf{x}}^{\mathrm{T}} \mathbf{P}_{1} \mathbf{B}\left[\mathbf{K}_{c}^{\mathrm{T}} \hat{\mathbf{x}}+\mathbf{u}+\mathbf{d}\right]+\tilde{\mathbf{x}}^{\mathrm{T}} \mathbf{P}_{1} \mathbf{f}(\mathbf{x})+\frac{1}{\gamma} \tilde{\mathbf{\Theta}}_{1} \dot{\tilde{\mathbf{\Theta}}}_{1}^{\mathrm{T}} \cdot(52)
\end{aligned}
$$

Using (44), (52) can be further written as

$$
\begin{aligned}
\dot{\mathrm{V}}= & -\frac{1}{2} \hat{\mathbf{x}}^{\mathrm{T}} \mathbf{Q} \hat{\mathbf{x}}-\frac{1}{2} \tilde{\mathbf{x}}^{\mathrm{T}} \mathbf{Q}_{1} \tilde{\mathbf{x}}+\hat{\mathbf{x}}^{\mathrm{T}} \mathbf{P} \mathbf{K}_{o} \mathbf{C}^{\mathrm{T}} \tilde{\mathbf{x}}+\tilde{\mathbf{x}}^{\mathrm{T}} \mathbf{P}_{1} \mathbf{f}(\mathbf{x}) \\
& +\tilde{\mathbf{x}}^{\mathrm{T}} \mathbf{P}_{1} \mathbf{B}\left[-\boldsymbol{\eta}_{1}(\tilde{\mathbf{x}}) \mathbf{\Theta}_{1} \xi(\hat{\mathbf{x}})-\mathbf{u}_{a}-\mathbf{u}_{R}-\mathbf{u}_{d}+\mathbf{d}\right] \\
& +\frac{1}{\gamma} \tilde{\boldsymbol{\Theta}}_{1} \dot{\tilde{\Theta}}_{1}^{\mathrm{T}} .
\end{aligned}
$$

By substituting the control signals, definition of $g_{1}(\mathbf{x}, \tilde{\mathbf{x}})$, $\hat{g}_{1}(\hat{\mathbf{x}})$, and $\eta_{1}^{\mathrm{T}}$, we have

$$
\begin{aligned}
\dot{V}= & -\frac{1}{2} \hat{\mathbf{x}}^{\mathrm{T}} \mathbf{Q} \hat{\mathbf{x}}-\frac{1}{2} \tilde{\mathbf{x}}^{\mathrm{T}} \mathbf{Q}_{1} \tilde{\mathbf{x}} \\
& +\left\|\boldsymbol{\eta}_{1}(\tilde{\mathbf{x}})\right\|^{2}\left(\boldsymbol{\Theta}_{1}^{*} \xi(\mathbf{x})-\boldsymbol{\Theta}_{1} \xi(\mathbf{x})+\delta_{n 1}\right)-k_{1}\left\|\boldsymbol{\eta}_{1}(\tilde{\mathbf{x}})\right\|^{2} \\
& +\tilde{\mathbf{x}}^{\mathrm{T}} \mathbf{P}_{1} \mathbf{B d}-k_{2} \tilde{\mathbf{x}}^{\mathrm{T}} \mathbf{P}_{1} \mathbf{B} \operatorname{sgn}\left(\mathbf{B}^{\mathrm{T}} \mathbf{P}_{1} \tilde{\mathbf{x}}\right)+\frac{1}{\gamma} \tilde{\boldsymbol{\Theta}}_{1} \dot{\tilde{\Theta}}_{1}^{\mathrm{T}} .
\end{aligned}
$$

By using Assumption 2 and adaptation law (48), the following relation is obtained:

$$
\begin{aligned}
\dot{\mathrm{V}} \leq & -\frac{1}{2} \hat{\mathbf{x}}^{\mathrm{T}} \mathbf{Q} \hat{\mathbf{x}}-\frac{1}{2} \tilde{\mathbf{x}}^{\mathrm{T}} \mathbf{Q}_{1} \tilde{\mathbf{x}}+\left(\delta_{n 1}-k_{1}\right)\left\|\boldsymbol{\eta}_{1}(\tilde{\mathbf{x}})\right\|^{2} \\
& +\left(\delta_{d}-k_{2}\right) \sum_{i=1}^{m}\left|\left(\tilde{\mathbf{x}}^{\mathrm{T}} \mathbf{P}_{1} \mathbf{B}\right)_{i}\right| .
\end{aligned}
$$

Now, if $k_{1} \geq\left\|\delta_{n 1}\right\|$ and $k_{2} \geq \delta_{d}$, we have

$$
\dot{\mathrm{V}} \leq-\frac{1}{2} \hat{\mathbf{x}}^{\mathrm{T}} \mathbf{Q} \hat{\mathbf{x}}-\frac{1}{2} \tilde{\mathbf{x}}^{\mathrm{T}} \mathbf{Q}_{1} \tilde{\mathbf{x}} .
$$


Denoting $\mathbf{Q}_{2}=\operatorname{diag}\left[\mathbf{Q}, \mathbf{Q}_{1}\right]$ and $\mathbf{X}^{\mathrm{T}}=\left[\hat{\mathbf{x}}^{\mathrm{T}}, \tilde{\mathbf{x}}^{\mathrm{T}}\right]$, becomes

$$
\dot{\mathrm{V}} \leq-\frac{1}{2} \mathbf{X}^{\mathrm{T}} \mathbf{Q}_{2} \mathbf{X}
$$

According to Barbalat lemma, it can be concluded that $\mathbf{x}, \hat{\mathbf{x}}, \mathbf{u} \in L_{\infty}$ and $\lim _{t \rightarrow \infty} \mathbf{X}=\mathbf{0}$. Thus, $\lim _{t \rightarrow \infty} \hat{\mathbf{x}}=\mathbf{0}$ and $\lim _{t \rightarrow \infty} \tilde{\mathbf{x}}=\mathbf{0}$. Since $\mathbf{x}=\hat{\mathbf{x}}+\tilde{\mathbf{x}}$ one can conclude that $\lim _{t \rightarrow \infty} \mathbf{x}=\mathbf{0}$ $t \rightarrow \infty$
which completes the proof.

Corollary 2: The weighs of NN in (48) eventually converge to constant values.

Proof: From Theorem 3, since $\lim _{t \rightarrow \infty}\|\tilde{\mathbf{x}}(t)\|=0$, using (38) and (48), one can conclude $\lim _{\rightarrow \infty} \dot{\Theta}_{1}=\mathbf{0}$. Consequently, the adaptation parameters of $\mathrm{NN} \stackrel{\rightarrow \infty}{\rightarrow}$ approach to constant values.

\section{Simulation Results}

In this section, the simulation study is carried out to show the proficiency of the proposed adaptive neural controller. Two numerical cases are considered to show the effectiveness of the proposed design. In addition, both state and observer-based controllers are presented for each case. The first case shows that the proposed methods can handle systems with $n>2 m$, while the second example manifests the method presented in this paper can address systems with $n \leq 2 m$.

\section{A. Example 1: System With $n>2 m$}

1) State-Feedback Design: The system considered here is a single-input nonlinear system obtained by adding a mismatched uncertain term to the system described in [28]. The resulting system can be written in terms of the following parameters:

$$
\begin{aligned}
\mathbf{A} & =\left[\begin{array}{lll}
-1 & 2 & 0 \\
1 & 0 & 3 \\
0 & 0 & 1
\end{array}\right], \mathbf{B}=\left[\begin{array}{l}
0 \\
0 \\
1
\end{array}\right] \\
\mathbf{f}(\mathbf{x}) & =\left(\begin{array}{c}
0.06 \sin (0.1 t) x_{2}+0.06 \sin (0.1 t) x_{3}+\sin \left(x_{3}\right) \\
1+0.03 \cos (0.1 t) x_{2}+0.03 \cos (0.1 t) x_{3}-\cos \left(x_{2}\right) \\
0.1 \cos (0.1 t) x_{3}+x_{1} x_{2}
\end{array}\right) \\
\mathbf{d} & =\sin (t) .
\end{aligned}
$$

The goal is to investigate stabilization of this system with the state-feedback stabilizer proposed in this paper. The design parameters for the state-feedback stabilizer are given by

$$
\begin{aligned}
& \mathbf{K}_{c}^{\mathrm{T}}=\left[\begin{array}{lll}
1.33 & 2.67 & 6
\end{array}\right] \\
& k_{1}=1, k_{2}=1.1, \gamma=5, \phi_{i}=0.1, \mathbf{Q}=\left[\begin{array}{lll}
5 & 0 & 0 \\
0 & 5 & 0 \\
0 & 0 & 5
\end{array}\right] .
\end{aligned}
$$

The vector gain $\mathbf{K}_{c}$ confirms that eigenvalues of the matrix $\mathbf{A}-\mathbf{B} \mathbf{K}_{c}^{\mathrm{T}}$ are assigned to $-1,-2$, and -3 . In this simulation, $l=9$ nodes are taken to construct RBFNN with centers evenly distributed in $\left[\begin{array}{lll}x_{1} & x_{2} & x_{3}\end{array}\right] \in\left[\begin{array}{ll}-4,4 & 4\end{array} \times[-4,4] \times[-4,4]\right.$, the widths are chosen as $\eta=1$, and the initial parameters of $\mathrm{NN}$ is a vector with constant value 0.1. The simulation is carried out with initial conditions $\mathbf{x}_{0}=\left[\begin{array}{lll}-1 & 3 & -2\end{array}\right]^{\mathrm{T}}$. The states of the closed-loop system using the proposed controller are illustrated in Fig. 2. As it can be seen in this figure, the
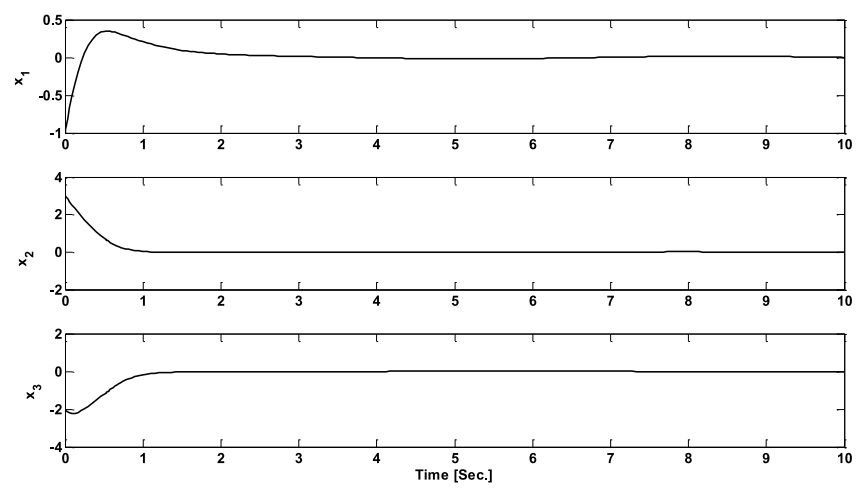

Fig. 2. State trajectories of the system by using state feedback adaptive-NN controller.

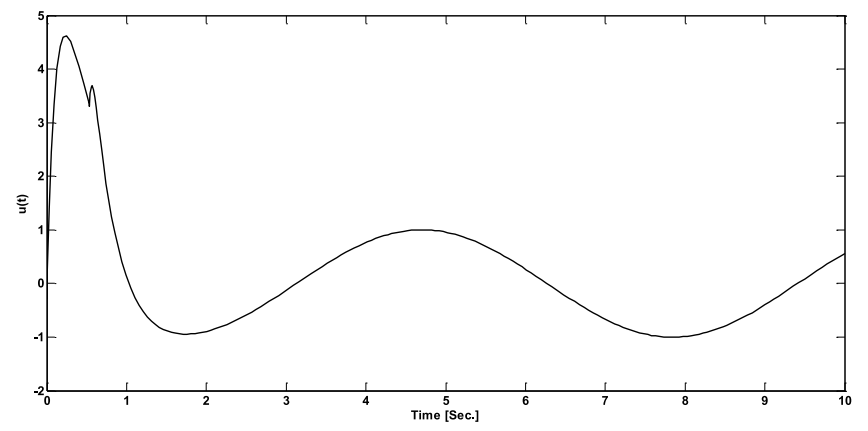

Fig. 3. Control input of the system by using state feedback adaptive-NN controller.

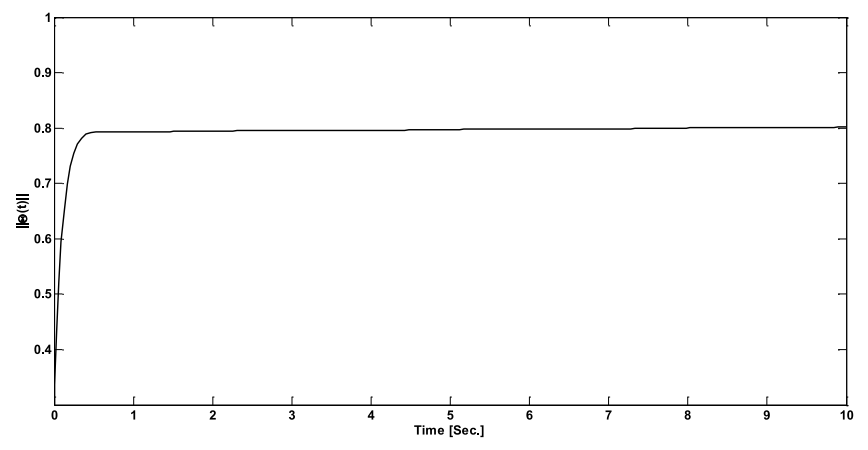

Fig. 4. Norm of weights of NN.

controller can effectively stabilize the system states in presence of mismatched uncertainties. Fig. 3 shows the control signal, illustrating the control signal is bounded. The controller shows the sinusoidal behavior in the steady-state response to compensate the effect of sinusoidal external disturbance.

Fig. 4 presents norm of weights of RBFNN. This figure shows that the parameters of $\mathrm{NN}$ ultimately converge to a constant value and remain bounded.

2) Observer-Based Stabilizer Design: In this part, it is assumed that state vector $\mathbf{x}$ is immeasurable. Therefore, an observer is to be designed for the estimation of the immeasurable states. Consider the system (32) with the parameters (58). Suppose the output matrix $\mathbf{C}^{\mathrm{T}}$ is given as follows:

$$
\mathbf{C}^{\mathrm{T}}=\left[\begin{array}{lll}
1 & 0 & 1
\end{array}\right] .
$$

The aim is to design a stabilizing controller by using measureable output. The following parameters are used 

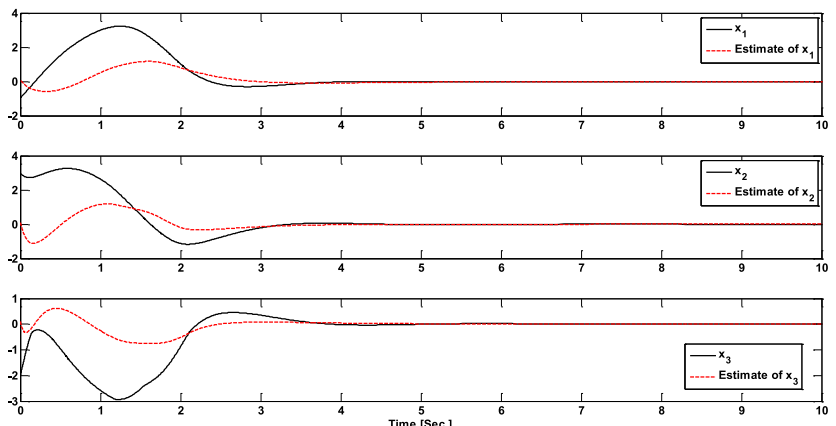

Fig. 5. True states and estimated states of the closed-loop system.

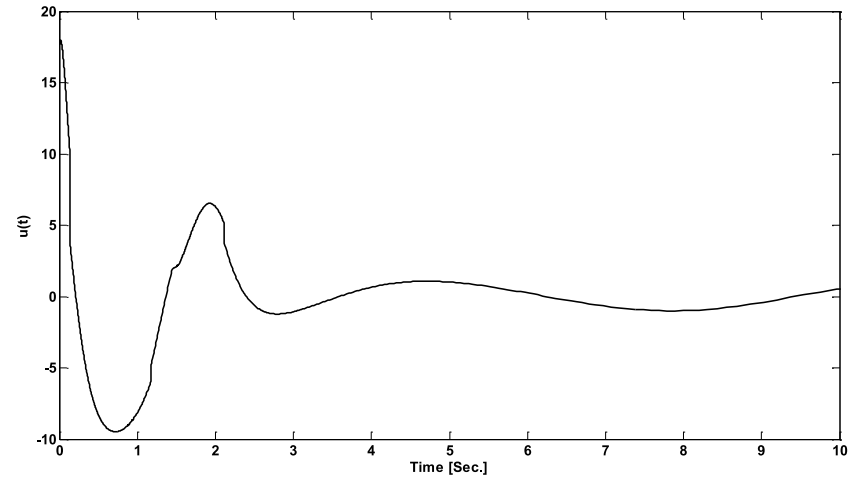

Fig. 6. Control signal of the system by using observer-based adaptive-NN controller.

to construct the stabilizing controller based on estimated states:

$$
\begin{aligned}
\mathbf{K}_{c}^{\mathrm{T}} & =\left[\begin{array}{lll}
1.33 & 2.67 & 6
\end{array}\right], \mathbf{K}_{o}=\left[\begin{array}{lll}
2 & 6 & 4
\end{array}\right]^{\mathrm{T}} \\
k_{1} & =5, k_{2}=3, \gamma=5, \phi_{i}=0.0001, \quad \mathbf{Q}=\left[\begin{array}{ccc}
0.1 & 0 & 0 \\
0 & 0.1 & 0 \\
0 & 0 & 0.1
\end{array}\right] .
\end{aligned}
$$

Using the vector gain $\mathbf{K}_{o}$, eigenvalues of the matrix $\mathbf{A}-\mathbf{K}_{o} \mathbf{C}^{\mathrm{T}}$ are assigned to $-1,-2$, and -3 . It can be shown that the matrices of system satisfy the conditions of Theorem 3. The simulation is carried out with initial conditions $\mathbf{x}_{0}=\left[\begin{array}{lll}-1 & 3 & -2\end{array}\right]^{\mathrm{T}}$ and $\hat{\mathbf{x}}_{0}=\left[\begin{array}{lll}0.1 & 0.1 & 0.1\end{array}\right]^{\mathrm{T}}$. In this simulation, we take $l=9$ nodes to construct the NN with centers evenly distributed in $\left[\hat{\mathbf{x}}_{1} \hat{\mathbf{x}}_{2} \hat{\mathbf{x}}_{3}\right] \in[-6,6] \times[-6,6] \times$ $[-6,6]$, the widths are chosen as $\eta=1.9$, and the initial parameters vector of $\mathrm{NN}$ is $\boldsymbol{\Theta}_{0}=\mathbf{0}$. Fig. 5 illustrates the states of the closed-loop system and corresponding estimated states. As seen in this figure, both states and estimated states are stabilized by using the observer-based controller. The control signal of the system depicts the smooth behavior as shown in Fig. 6.

Example 1 showed that the proposed methods are applicable for nonlinear systems with $n>2 m$. The second example is provided to investigate the capability of the presented scheme for systems with $n \leq 2 m$.

\section{B. Example 2: System With $n \leq 2 m$}

1) State-Feedback Design: Consider again nonlinear system (1) with mismatched uncertainties and external

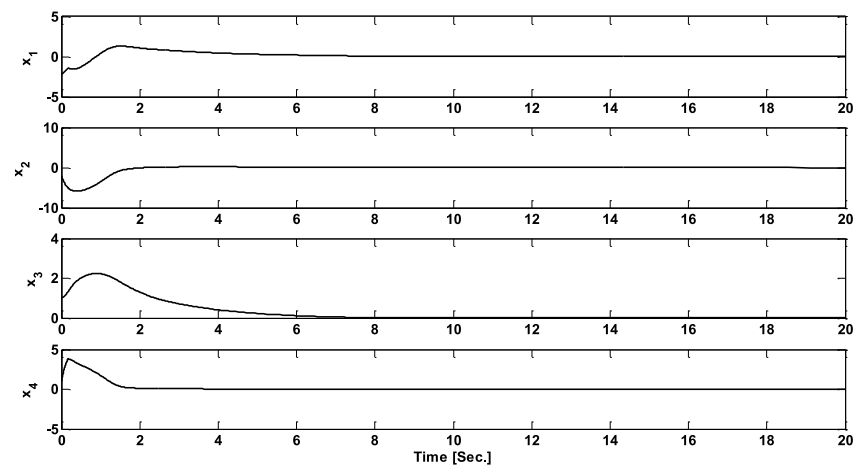

Fig. 7. States of the system by using state feedback adaptive-NN controller.

disturbance. Define a system with two control inputs and the following parameters:

$$
\begin{aligned}
\mathbf{A} & =\left[\begin{array}{cccc}
-0.0366 & 0.0271 & 0.0188 & -0.4555 \\
0.0482 & -1.01 & 0.0024 & -4.0208 \\
0.1002 & 0.3681 & -0.7070 & 1.42 \\
0 & 0 & 1 & 0
\end{array}\right], \mathbf{B}=\left[\begin{array}{cc}
1 & -1 \\
0 & -1 \\
0 & 0 \\
1 & 0
\end{array}\right] \\
\mathbf{f}(\mathbf{x})= & \left(\begin{array}{c}
\left(0.05 x_{3}+0.1 x_{4}\right) \sin \left(0.1 x_{2}\right)+x_{3}+2 \cos \left(0.2 x_{1}\right) x_{2} \\
-0.05 x_{4} \cos \left(0.3 x_{4} t\right)+2 \cos \left(0.2 x_{1}\right) x_{2} \\
-0.2 x_{1}-0.05 x_{2} \\
\left(0.2 x_{1}+0.05 x_{4}\right) \sin \left(0.4 x_{3}\right)+x_{3}
\end{array}\right) .
\end{aligned}
$$

The following parameters are chosen to design the statefeedback stabilizer:

$$
\begin{aligned}
\mathbf{K}_{c}^{\mathrm{T}} & =\left[\begin{array}{cccc}
7.1045 & -6.7095 & -12.4610 & -1.8744 \\
15.2630 & -18.2794 & -37.2436 & -12.2484
\end{array}\right] \\
k_{1} & =2, k_{2}=6, \gamma=1, \phi_{i}=10^{-4} \\
\mathbf{Q} & =\left[\begin{array}{cccc}
2.5 & 0 & 0 & 0 \\
0 & 2.5 & 0 & 0 \\
0 & 0 & 2.5 & 0 \\
0 & 0 & 0 & 2.5
\end{array}\right] .
\end{aligned}
$$

The simulation is carried out with initial conditions $\mathbf{x}_{0}=$ $\left[\begin{array}{llll}-2 & -2 & 1 & 1\end{array}\right]^{\mathrm{T}}$. In this simulation, $l=9$ nodes are taken to construct the RBFNN with centers evenly distributed in $\left[\begin{array}{llll}x_{1} & x_{2} & x_{3} & x_{4}\end{array}\right] \in[-2,2] \times[-2,2] \times[-2,2] \times[-2,2]$, the widths are chosen as $\eta=1.9$, and the initial parameters of $\mathrm{NN}$ is a random vector generated in the MATLAB environment. Fig. 7 manifests states of the closed-loop using the presented controller. This figure shows that in the presence of mismatched uncertainties and external disturbances, the proposed controller can effectively regulate system states. The control signals used for stabilization are illustrated in Fig. 8. This figure shows that in order to alleviate the effects of external disturbances, the controller has sinusoidal and constant waveform for the first and second inputs in the steady-state response, respectively.

2) Observer-Based Stabilizer Design: Let us consider again the previous example with the following output matrix:

$$
\mathbf{C}=\mathbf{B} \text {. }
$$



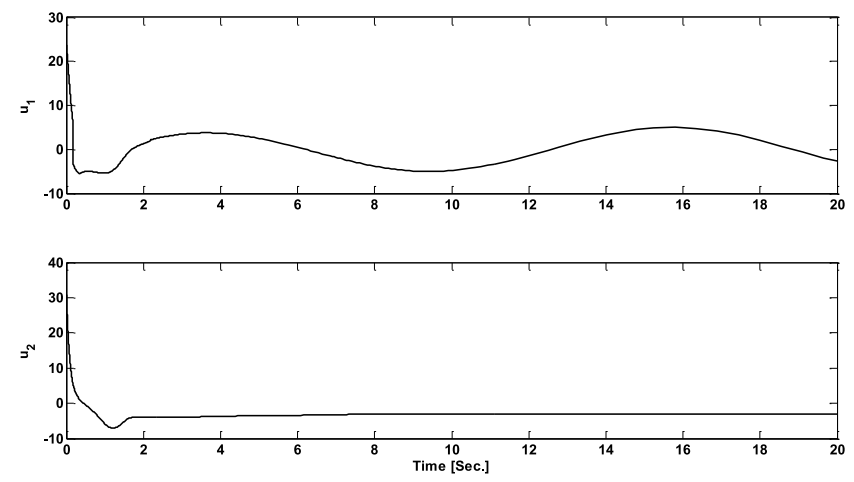

Fig. 8. Control inputs of the system generated by state feedback adaptive-NN controller.

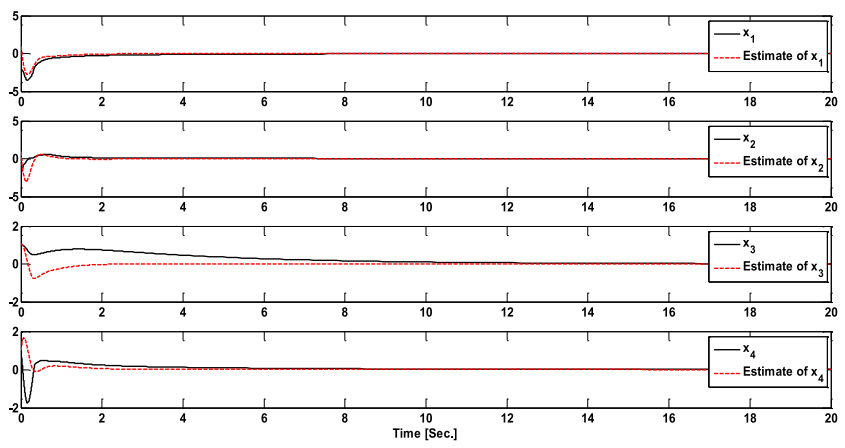

Fig. 9. True states and estimated states of the closed-loop system.
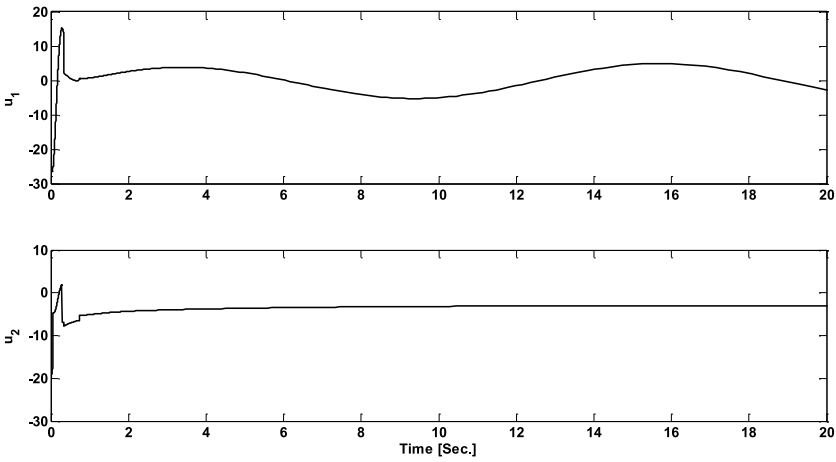

Fig. 10. Control inputs of the system by using observer-based adaptive-NN controller.

The following parameters are used to construct the stabilizing controller based on estimated states:

$$
\begin{aligned}
& \mathbf{K}_{c}^{\mathrm{T}}=\left[\begin{array}{cccc}
7.1045 & -6.7095 & -12.4610 & -1.8744 \\
15.2630 & -18.2794 & -37.2436 & -12.2484
\end{array}\right] \\
& \mathbf{K}_{o}=\left[\begin{array}{cccc}
0.0144 & -3.8088 & 1.9378 & 2.9193 \\
-1.8687 & -3.4440 & 1.3491 & 1.9959
\end{array}\right]^{\mathrm{T}} \\
& k_{1}=3, k_{2}=6, \gamma=0.5, \phi_{i}=10^{-4} \\
& \mathbf{Q}=\left[\begin{array}{cccc}
0.25 & 0 & 0 & 0 \\
0 & 0.25 & 0 & 0 \\
0 & 0 & 0.25 & 0 \\
0 & 0 & 0 & 0.25
\end{array}\right]
\end{aligned}
$$

It can be shown that the matrices of system satisfy the conditions of Theorem 3. The simulation is carried out with initial conditions $\mathbf{x}_{0}=\left[\begin{array}{llll}-2 & -2 & 1 & 1\end{array}\right]^{\mathrm{T}}$ for the system, and $\hat{\mathbf{x}}_{0}=$ $\left[\begin{array}{llll}1 & 1 & 1 & 1\end{array}\right]^{\mathrm{T}}$ for the observer. In this simulation, we take $l=21$ nodes to construct the $\mathrm{NN}$ with centers evenly distributed in $\left[\hat{x}_{1} \hat{x}_{2} \hat{x}_{3} \hat{x}_{4}\right] \in[-4,4] \times[-4,4] \times[-4,4] \times[-4,4]$, the widths are chosen as $\eta=1.2$, and the initial parameters of $\mathrm{NN}$ is a random vector. Fig. 9 illustrates the states of the closedloop system and the corresponding estimated states. As seen in this figure, both states and estimated states are stabilized by using the observer-based controller. The control signals of the system show the smooth behavior as depicted in Fig. 10.

\section{CONCLUSION}

In this paper, two state-feedback and observer-based adaptive neural network controllers for stabilization of nonlinear systems with mismatched uncertainties were presented. By using an RBFNN, the bound of unknown nonlinear functions is approximated so that no information about the upper bound of mismatched uncertainties is required. The state-feedback and observer-based controllers are based on Lyapunov and SPR-Lyapunov stability theorem, respectively, and it was shown that the asymptotic convergence of the closed-loop system to zero is achieved while maintaining bounded states at the same time. The presented methods can handle both systems with $n \leq 2 m$ and $n>2 m$, where $\mathrm{n}$ and $\mathrm{m}$ are the number of system states and control inputs, respectively. Simulation results reveal the effectiveness of the given methods in the stabilization of nonlinear systems with mismatched uncertainties. Although this paper addressed the system stabilization with mismatched uncertainties, other restrictive and challenging phenomena like time-delay and variable structure systems with mismatched uncertainties can be further studied in the future.

\section{ACKNOWLEDGMENT}

The author, H. R. Karimi, would like to thank the support of Deutscher Akademischer Austauschdienst (DAAD) program in this paper.

\section{REFERENCES}

[1] A. Isidori, Nonlinear Control Systems, 3rd ed. Berlin, Germany: Springer Verlag, 1995

[2] M. Wang, X. Liu, and P. Shi, "Adaptive neural control of pure-feedback nonlinear time-delay systems via dynamic surface technique," IEEE Trans. Syst., Man, Cybern. B, Cybern., vol. 41, no. 6, pp. 1681-1692, Dec. 2011.

[3] R. Marino and P. Tomei, Nonlinear Control Design: Geometric, Adaptive and Robust. London, U.K.: Prentice Hall, 1995.

[4] M. M. Arefi and M. R. Jahed-Motlagh, "Adaptive robust synchronization of Rossler systems in the presence of unknown matched time-varying parameters," Commun. Nonlinear Sci. Numer. Simul., vol. 15, no. 12, pp. 4149-4157, 2010.

[5] R. Marino and P. Tomei, "Robust stabilization of feedback linearizable time-varying uncertain nonlinear systems," Automatica, vol. 29, no. 1, pp. 181-189, 1993.

[6] M. M. Polycarpou and P. A. Ioannou, "A robust adaptive nonlinear control design," Automatica, vol. 32, no. 3, pp. 423-427, 1996.

[7] Y. Hashimoto, "Robust output tracking of nonlinear systems with mismatched uncertainties," Int. J. Control, vol. 72, no. 5, pp. 411-417, 1999.

[8] M. M. Arefi and M. R. Jahed-Motlagh, "Adaptive robust stabilization of a class of uncertain non-linear systems with mismatched time-varying parameters," J. Syst. Control Eng., vol. 226, no. 2, pp. 204-214, 2012.

[9] S. N. Singh and A. A. R. Coelho, "Nonlinear control of mismatched uncertain linear systems and application to control of aircraft," J. Dyn. Syst. Meas. Control, vol. 106, no. 3, pp. 203-210, 1984. 
[10] C. C. Wen and C. C. Cheng, "Design of sliding surface for mismatched uncertain systems to achieve asymptotical stability," J. Franklin Inst., vol. 345, no. 8, pp. 926-941, 2008.

[11] V. Utkin, "Variable structure systems with sliding modes," IEEE Trans. Autom. Control, vol. 22, no. 2, pp. 212-222, Apr. 1977.

[12] C. H. Chou and C. C. Cheng, "A decentralized model reference adaptive variable structure controller for large-scale time-varying delay systems," IEEE Trans. Autom. Control, vol. 48, no. 7, pp. 1213-1217, Jul. 2003.

[13] Y. Jun, L. Shihua, and Y. Xinghuo, "Sliding-mode control for systems with mismatched uncertainties via a disturbance observer," IEEE Trans. Ind. Electron., vol. 60, no. 1, pp. 160-169, Jan. 2013.

[14] H. K. Khalil, Nonlinear Systems, 3rd ed. Upper Saddle River, NJ, USA: Prentice Hall, 2002.

[15] J. J. Slotine and W. Li, Applied Nonlinear Control. Englewood Cliffs, NJ, USA: Prentice-Hall, 1991.

[16] F. Castanos and L. Fridman, "Analysis and design of integral sliding manifolds for systems with unmatched perturbations," IEEE Trans. Autom. Control, vol. 51, no. 5, pp. 853-858, May 2006.

[17] Z. Liu, H. Su, and S. Pan, "Passivity-based adaptive integral sliding mode control of uncertain nonlinear systems," in Proc. 8th World Congr. Intell. Control Autom., Jinan, China, 2010, pp. 3939-3944.

[18] M. L. Chan, C. W. Tao, and T. T. Lee, "Sliding mode controller for linear systems with mismatched time-varying uncertainties," J. Franklin Inst., vol. 337, nos. 2-3, pp. 105-115, 2000

[19] J. X. Xu, Y. J. Pan, and T. H. Lee, "Analysis and design of integral sliding mode control based on Lyapunov's direct method," in Proc. Amer Control Conf., Denver, CO, USA, 2003, pp. 192-196.

[20] A. C. Huang and Y. C. Chen, "Adaptive multiple-surface sliding control for non-autonomous systems with mismatched uncertainties," Automatica, vol. 40, no. 11, pp. 1939-1945, 2004.

[21] W. J. Cao and J. X. Xu, "Nonlinear integral-type sliding surface for both matched and unmatched uncertain systems," IEEE Trans. Autom. Control, vol. 49, no. 8, pp. 1355-1360, Aug. 2004.

[22] Y. W. Tsai, K. K. Shyu, and K. C. Chang, "Decentralized variable structure control for mismatched uncertain large-scale systems: A new approach," Syst. Control Lett., vol. 43, no. 2, pp. 117-125, 2005.

[23] C. Lin, Q. G. Wang, and T. H. Lee, "Stabilization of uncertain fuzzy time-delay systems via variable structure control approach," IEEE Trans. Fuzzy Syst., vol. 13, no. 6, pp. 787-798, Dec. 2005

[24] H. H. Choi, "LMI-based sliding surface design for integral sliding mode control of mismatched uncertain systems," IEEE Trans. Autom. Control, vol. 52, no. 4, pp. 736-742, Apr. 2007.

[25] H. H. Choi, "An LMI-based switching surface design method for a class of mismatched uncertain systems," IEEE Trans. Autom. Control, vol. 48, no. 9, pp. 1634-1638, Sep. 2003.

[26] H. H. Choi and K. S. Ro, "LMI-based sliding-mode observer design method," IEE Proc. Control Theory Appl., vol. 152, no. 1, pp. 113-115, 2005.

[27] K. S. Kim, Y. Park, and S. H. Oh, "Designing robust sliding hyperplanes for parametric uncertain systems: A Riccati approach," Automatica, vol. 36, no. 7, pp. 1041-1048, 2000 .

[28] C. W. Tao, C. M. Lang, and L. T. Tian, "Adaptive fuzzy sliding mode controller for linear systems with mismatched time-varying uncertainties," IEEE Trans. Syst., Man, Cybern. B, Cybern., vol. 33, no. 2, pp. 283-294, Apr. 2003.

[29] H. R. Karimi and H. Gao, "New delay-dependent exponential H $\infty$ synchronization for uncertain neural networks with mixed time delays," IEEE Trans. Syst., Man, Cybern. B, Cybern., vol. 40, no. 1, pp. 173-185, Feb. 2010.

[30] C. C. Cheng, C. C. Wen, and S. P. Chen, "Design of adaptive output feedback variable structure tracking controllers," JSME Int. J. Series C Mech. Syst. Mach. Elements Manuf., vol. 49, no. 2, pp. 432-437, 2006.

[31] K. K. Shyu, Y. W. Tsai, and C. K. Lai, "Sliding mode control for mismatched uncertain systems," Electron. Lett., vol. 34, no. 24, pp. 2359-2360, 1998

[32] H. H. Choi, "On the existence of linear sliding surfaces for a class of uncertain dynamic systems with mismatched uncertainties," Automatica, vol. 35 , no. 10 , pp. $1707-1715,1999$.

[33] Y. Chang and C. C. Cheng, "Design of adaptive sliding surfaces for systems with mismatched perturbations to achieve asymptotical stability," IET J. Control Theory Appl., vol. 1, no. 1, pp. 417-421, 2007.
[34] Y. Chang and C. C. Cheng, "Adaptive sliding mode control for plants with mismatched perturbations to achieve asymptotical stability," Int. J. Robust Nonlinear Control, vol. 17, no. 9, pp. 880-896, 2007.

[35] C. C. Cheng and C. Z. Guo, "Design of adaptive sliding mode controllers for systems with mismatched uncertainty to achieve asymptotic stability," in Proc. Amer. Control Conf., Baltimore, MD, USA, 2010, pp. $1683-1688$.

[36] C. C. Cheng, G. L. Su, and C. W. Chien, "Block backstepping controllers design for a class of perturbed non-linear systems with M blocks," IET J. Control Theory Appl., vol. 6, no. 13, pp. 2021-2030, 2012.

[37] M. M. Arefi and M. R. Jahed-Motlagh, "Observer-based adaptive neural control of uncertain MIMO nonlinear systems with unknown control direction," Int. J. Adapt. Control Signal Process., vol. 27, no. 9, pp. 741-754, 2013.

[38] Y. H. Chang and W.-S. Chan, "Adaptive dynamic surface control for uncertain nonlinear systems with interval type-2 fuzzy neural networks," IEEE Trans. Cybern., vol. 44, no. 2, pp. 293-304, Feb. 2014.

[39] R. Hedjar, "Adaptive neural network model predictive control," Int. J. Innov. Comput., Inf. Control, vol. 9, no. 3, pp. 1245-1257, 2013.

[40] H. Wang, B. Chen, and C. Lin, "Adaptive neural tracking control for a class of stochastic nonlinear systems with unknown dead-zone," Int. J. Innov. Comput., Inf. Control, vol. 9, no. 8, pp. 3257-3269, 2013.

[41] M. Chen and S. S. Ge, "Direct adaptive neural control for a class of uncertain nonaffine nonlinear systems based on disturbance observer," IEEE Trans. Cybern., vol. 43, no. 4, pp. 1213-1225, Aug. 2013.

[42] H. Du and X. Chen, "NN-based output feedback adaptive variable structure control for a class of non-affine nonlinear systems: A nonseparation principle design," Neurocomputing, vol. 72, nos. 7-9, pp. 2009-2016, 2009.

[43] M. M. Arefi, Z. Ramezani, and M. R. Jahed-Motlagh, "Observerbased adaptive robust control of nonlinear nonaffine systems with unknown gain sign,” Nonlinear Dyn., pp. 1-10, Jul. 2014, DOI: $10.1007 / \mathrm{s} 11071-014-1573-0$.

[44] M. M. Arefi, J. Zarei, and H. R. Karimi, "Adaptive output feedback neural network control of uncertain non-affine systems with unknown control direction," J. Franklin Inst., vol. 351, no. 8, pp. 4302-4316, 2014.

[45] J. Qiu, G. Feng, and J. Yang, "A new design of delay-dependent robust $\mathrm{H} \infty$ filtering for discrete-time T-S fuzzy systems with timevarying delay," IEEE Trans. Fuzzy Syst., vol. 17, no. 5, pp. 1044-1058, Oct. 2009

[46] J. Qiu, G. Feng, and H. Gao, "Fuzzy-model-based piecewise $\mathrm{H} \infty$ staticoutput-feedback controller design for networked nonlinear systems," IEEE Trans. Fuzzy Syst., vol. 18, no. 5, pp. 919-934, Oct. 2010.

[47] X. Su, P. Shi, L. Wu, and Y. D. Song, "A novel control design on discrete-time Takagi-Sugeno fuzzy systems with time-varying delays," IEEE Trans. Fuzzy Syst., vol. 21, no. 4, pp. 655-671, Aug. 2013.

[48] S. Bououden, M. Chadli, and H. R. Karimi, "Fuzzy sliding mode controller design using Takagi-Sugeno modelled nonlinear systems," Math. Probl. Eng., vol. 2013, pp. 1-7, Feb. 2013.

[49] H. R. Karimi and M. Chadli, "Robust observer design for Takagi-Sugeno fuzzy systems with mixed neutral and discrete delays and unknown inputs," Math. Probl. Eng., vol. 2012, pp. 1-13, Jan. 2012.

[50] L. Wu, X. Su, and P. Shi, "Output feedback control of Markovian jump repeated scalar nonlinear systems," IEEE Trans. Autom. Control, vol. 59, no. 1, pp. 199-204, Jan. 2014.

[51] R. D. Nussbaum, "Some remarks on a conjecture in parameter adaptive control," Syst. Control Lett., vol. 3, no. 5, pp. 243-246, 1983.

[52] S. M. Hoseini, M. Farrokhi, and A. J. Koshkouei, "Robust adaptive control of nonlinear non-minimum phase systems with uncertainties," Automatica, vol. 47, no. 2, pp. 348-357, 2011.

[53] H. L. Li, X. Jing, and H. R. Karimi, "Output-feedback based $\mathrm{H} \infty$ control for active suspension systems with control delay," IEEE Trans. Ind. Electron., vol. 61, no. 1, pp. 436-446, Jan. 2014.

[54] J. Rubió-Massegú, J. M. Rossell, H. R. Karimi, and F. Palacios-Quiñonero, "Static output-feedback control under information structure constraints," Automatica, vol. 49, no. 1, pp. 313-316, 2013.

[55] G. Strang, Introduction to Applied Mathematics. Wellesley, MA, USA: Wellesley-Cambridge Press, 1986.

[56] M. Corless and R. Shorten, "On a class of generalized eigenvalue problems and equivalent eigenvalue problems that arise in systems and control theory," Automatica, vol. 47, no. 3, pp. 431-442, 2011. 